\title{
RESEARCH
}

\section{research note: studying a new phase of europeanisation of national parliaments}

\author{
katjana gattermann $^{a, ~ *}$, anna-lena högenauer ${ }^{b}$ and \\ ariella huff ${ }^{c}$
}

${ }^{a}$ Amsterdam Centre for Contemporary European Studies (ACCESS EUROPE), Department of Political Science and Public Administration, Vrije Universiteit Amsterdam, De Boelelaan 1081, Amsterdam 1081 HV, The Netherlands

E-mail: k.gattermann@uva.nl

${ }^{b}$ Institute of Political Science, University of Luxembourg, Route de Diekirch,

Walferdange 7220, Luxembourg

E-mail: anna-lena.hoegenauer@uni.lu

${ }^{\mathrm{C}}$ Foreign Affairs Committee, Committee Office, House of Commons, London

SW1A 0AA, UK

E-mail: huffa@parliament.uk

${ }^{*}$ Corresponding author.

doi:10.1057/eps.2015.56; published online 16 October 2015

\begin{abstract}
In this research note, we propose studying a new trend of Europeanisation in national parliaments within the European Union (EU). We argue that further integration, combined with the opportunities and challenges presented by the Lisbon Treaty and the financial crisis, created pressure on national parliaments to expand the scrutiny process beyond European Affairs Committees. In this new phase of Europeanisation, parliaments are increasingly 'mainstreaming' EU affairs scrutiny, blurring the distinction between national and European policies and involving larger numbers of MPs. Following a review of existing research on the Europeanisation of national parliaments in the post-Lisbon era, we propose studying four dimensions of mainstreaming: the rising involvement of sectoral committees in European affairs; the adaptation of parliamentary staff to EU policy-making; the growing salience of European affairs in plenary debates; and increasing inter-parliamentary cooperation beyond European affairs specialists. We argue that this trend has significant implications for research that studies the roles of national parliaments in the democratic functioning of the EU.
\end{abstract}

Keywords national parliaments; Europeanisation; legislative studies; European Union studies; comparative politics 
$\mathrm{N}$ ational parliaments have traditionally struggled to scrutinise European Union (EU) affairs effectively. The erosion of the unanimity principle in the Council of Ministers and informational imbalances between national executives and legislatures have further complicated effective control over national executives (Norton, 1996; O'Brennan and Raunio, 2007). National parliaments themselves have only started to counteract these developments relatively late in the process, and many parliaments initially proved either unwilling or unable to gain a measure of influence over their governments (Maurer and Wessels, 2001).

From the early 2000 s onwards, scholarship focused on the Europeanisation of national parliaments, that is, the topdown impact of European integration on the functioning of parliaments ${ }^{1}$ (e.g., Raunio and Hix, 2000; Dimitrakopoulos, 2001; Auel and Benz, 2005; Raunio and Wiberg, 2009). This literature has tended to emphasise parliaments' efforts and adaptation to combat the deparliamentarisation phenomenon, with Winzen, for example, arguing that the strength of national parliaments overall increased from 2000 to 2010 (Winzen, 2012: 663$65){ }^{2}$ Many of these studies focused in particular on the role of European Affairs Committees (EACs) in empowering parliaments to scrutinise EU affairs (e.g., Dimitrakopoulos, 2001; Bergman et al, 2003; Auel, 2005).

However, we have recently observed changes in the organisation of EU affairs scrutiny, which suggest that a second phase of Europeanisation of national parliaments has started: one that sees a diffusion of European affairs scrutiny responsibilities to a wider range of actors away from their centralisation in EU affairs committees. This trend is called 'decentralisation' by Dutch practitioners (van Keulen, 2012; Tweede Kamer, 2006: 23-24) and 'mainstreaming' in the UK context (Carter and McLeod, 2006; European Scrutiny Committee, House of Commons, 2013). 'Mainstreaming' refers to the idea that a certain type of policy (EU affairs) is not to be treated in isolation, but is increasingly integrated into the work of parliaments in all policy sectors. We argue that it concerns all types of parliamentary actors, including parliamentary committees, political parties, individual legislators and support staff. However, the concept of mainstreaming is not yet subject to research on the Europeanisation of national parliaments.

In this research note, we argue that there is considerable pressure for mainstreaming, which necessitates the study of this new process in legislative research. In the first section, we discuss the factors that have led to this trend, as well as possible explanations for the variation in the speed and extent to which parliaments mainstream. In the second section, we provide an overview of recent research on the Europeanisation of national parliaments and derive implications for the study of mainstreaming. We argue that the trend towards mainstreaming affects in particular four dimensions for parliamentary scrutiny - the involvement of different types of committees, participation in inter-parliamentary cooperation (IPC), the organisation of staff support and participation in plenary debates - which are then discussed in the context of the existing empirical evidence. The final section discusses the implications of this trend for future research on the Europeanisation of national parliaments.

\section{THE PRESSURES FOR MAINSTREAMING}

For decades, the trend among parliaments has been to delegate the scrutiny 
of EU affairs to a clearly defined group of people represented in EACs (Raunio, 1999). So why are we now observing a trend towards mainstreaming? The change in trends can best be understood through a historical institutionalist approach that perceives the trend towards mainstreaming as a process, situating different parliaments at varying stages of that process in ways that reflect their particular domestic contexts. It relies on a broad definition of institutions that encompasses both formal rules and structures and informal but widely accepted practices (Rosamond, 2000: 114; Hall and Taylor, 1996: 938). This breadth is particularly useful in the study of national parliaments, enabling us to refer not only to the formal powers and roles assigned to them by national constitutions, but also to the political parties and individual parliamentarians operating within them and to the informal practices embedded in their daily operations.

In light of this framework, we can identify three main external pressures for spreading European affairs scrutiny outside the confines of specialised EACs that create a turning point for national parliaments. The first and longest-term major driver of mainstreaming derives from the general trend towards greater EU involvement in an ever-larger number of policy areas. This was expanded and codified in the Treaty of Lisbon, but has its roots in a broader and more gradual process that began to accelerate with the Single European Act. Most aspects of domestic policy now have a European dimension, meaning that the distinction between 'domestic' and 'European' policies has become blurred. For parliaments, effective scrutiny of EU affairs now requires the mobilisation of expertise across an ever-larger range of issues and policy areas. This puts the capacity of EACs to deal with all EU matters under pressure.

\section{'So why are we now observing a trend} towards mainstreaming?'

The second, more proximate, pressure for mainstreaming derives from the Treaty of Lisbon's provisions on enhancing the roles of national parliaments, especially the Early Warning System (EWS). If national parliaments feel that an EU legislative proposal breaches the principle of subsidiarity, they can now issue reasoned opinions to put the Commission under pressure to revise or withdraw the proposal. However, issuing reasoned opinions to the Commission, coordinating with other parliaments in the EWS and coping with the volume of information that parliaments now receive on EU affairs requires a significant commitment of resources, including both time and administrative support. This again puts a great strain on the capacity of EACs to scrutinise European affairs on their own.

Finally, from 2008 onwards, the global financial crisis, the Eurozone crisis and the measures taken to combat them have also significantly increased the political salience of the EU in national parliaments, particularly within the Eurozone. Questions regarding 'bailout' packages for struggling countries have become paramount in both rescued countries and rescuers, as austerity budgets have drawn protest in parts of Europe and support in others. As a result, the distinction between 'national' and 'European' politics has become ever more blurred, with even mainstream parties - as opposed to explicitly Eurosceptic groups - fighting elections with explicit reference to European-level issues (e.g., Francois Hollande's promise to re-negotiate the fiscal compact). The need to slash budgets to comply with EU rules even caused the 
collapse of the Dutch government in 2012 , striking at the heart of supposedly 'domestic' political life. In this context, there is pressure for a larger number of parliamentarians to be aware of EU issues in order to respond to the concerns of their constituents.

Moreover, the willingness and ability of national parliaments to mainstream EU affairs scrutiny are heavily conditioned by their existing procedures, resources, party-political dynamics and institutional cultures. Historical institutionalism, in particular, allows for a temporal dimension that can perceive the trend towards mainstreaming as a process, situating different parliaments at varying stages of that process in ways that reflect their particular domestic contexts. The broad definition of institutions employed by historical institutionalism allows it to draw on both a 'calculus approach' - that is, a rational-choice paradigm - and a 'cultural approach' emphasising the so-called 'logic of appropriateness' to understanding actor behaviour in the context of institutions (Rosamond, 2000: 114; Hall and Taylor, 1996: 938; Armstrong and Bulmer, 1998).

The first disincentive to mainstreaming relates to cost, in terms of both resources and time. Sectoral committees in many countries already consider themselves to have a full workload, and are likely to resent being asked to take on additional responsibilities unless accompanied by significant increases in resources. For example, van Keulen (2012) has highlighted that a major lesson of the Dutch case - one of the pioneers of mainstreaming - has been that sectoral committees have required both training and support for mainstreaming to work effectively. Mainstreaming also demands extensive cooperation between committees and groups of engaged parliamentarians (MPs) - between the EAC and sectoral committees, for example. Especially in the early phases, EACs may be reluctant to cede competences to other committees, as this may affect their status.

A second factor influencing the relative speed with which parliaments choose to mainstream scrutiny relates to the political salience of EU issues. This may be affected by two elements. Although the financial crisis has generally increased the visibility of EU affairs (see Saurugger, 2014), there remain several countries in which the effect of the crisis has been more limited - for example, because they are outside the Eurozone (e.g., the United Kingdom). In such cases, we expect the pressure for mainstreaming to be less in 'creditor' states like Germany or (former) 'debtor' states like Ireland and Greece, where the Eurozone crisis has permeated almost all aspects of national policy, including domestic party politics. In addition, the degree of pro-Europeanness of important MPs may play a role, as scrutiny of EU affairs may be regarded as unnecessary or because the EU remains overwhelmingly popular and thus is not seen as deserving of significant scrutiny (Raunio, 1999: 190).

The final major disincentive to mainstreaming relates specifically to parliaments in which existing scrutiny procedures, though centralised in the EAC, are already perceived as highly effective. The Danish Folketing, for example, is regularly cited as one of the EU's 'strongest' parliaments; scrutiny work there remains largely concentrated in the EAC (Winzen, 2012: 666). In cases like these, where the parliament in question considers itself to be very effective in controlling and monitoring its government and European legislation despite the relative lack of involvement of sectoral committees, we expect the costs of reform to outweigh the perceived benefits, at least for the foreseeable future. By contrast, mainstreaming will be easier to achieve when a parliament seeks to play a stronger role in EU affairs but is 
dissatisfied with the current committee system, which was, for example, the case in the Netherlands after the failed referendum on the Constitutional Treaty (Högenauer, 2015).

\section{RESEARCH TRENDS IN THE FIELD OF EUROPEANISATION OF NATIONAL PARLIAMENTS}

The phenomenon of mainstreaming has thus far not been subject to any study that investigates EU affairs scrutiny by national parliaments. Europeanisation is nevertheless a prominent theme. Inspired by meta-analyses in the field of Europeanisation (e.g., Machill et al, 2006; Exadaktylos and Radaelli, 2009), we provide a qualitative analysis of trends in research on the Europeanisation of national parliaments. For this, we selected twenty-two studies through searching the Web of Science and Google Scholar by the keywords 'Europeani\$ation' and 'national parliaments'. In order to be selected, studies had to be published in English-speaking, peer-reviewed journals in the field of political science or public administration. Peer review ensures high quality and articles in English infer a high reach within the academic community. We decided that (parts of) the time period of investigation had to be post-Lisbon in order to identify recent trends and thus focus on articles that have been published between 1 January 2010 and 31 July 2015. Furthermore, we disregarded theoretical reflections or literature reviews and solely selected explicitly empirical studies. The empirical approach had to be systematic and the research design comparative in order for us to draw inferences about Europeanisation trends across national parliaments.

Table 1 provides an overview of the selected studies. We have identified the empirical focus of each study, their method(s) of analysis, as well as the parliaments under study and the time period of investigation. Eight studies comprise national parliaments of all EU-27 member states, while the rest deal with a subselection of national parliaments, ranging from two to twenty-six chambers. Not least because of our selection criteria, we observe that two-thirds of the studies explicitly respond to two of the main external pressures that we discussed above, that is, either the new provisions by the Lisbon Treaty (eight articles) or the Eurozone crisis (three articles); three studies acknowledge both pressures. Furthermore, following our argumentation above (that EU affairs cannot be treated in isolation anymore given the interlinkage of domestic and European policy areas), we notice the breadth of policy areas covered by the studies: several articles represent case studies related to a variety of EU legislation (e.g., Foreign and Security Policy, migration law) or decision-making processes (e.g., regarding the first yellow card in the EWS, EU budgets), while others take a more inclusive approach as regards parliamentary activity and scrutiny.

The articles are rather diverse in terms of empirical focus. Most articles deal with more specific forms of parliamentary scrutiny, such as by the issuing of reasoned opinions, mandates or resolutions as well as committee referrals or debates (eleven articles), while four articles take a broader perspective of parliamentary oversight (Dörrenbächer et al, 2015; Huff, 2015; Jensen and Martinsen, 2015; Peters et al, 2014) and Strelkov (2015) investigates the relationships between parliamentary committees, parties and administrators in EU affairs scrutiny. Inter-parliamentary cooperation and coordination as well as the role of parliamentary administrations are subject to four articles. Lastly, two articles propose indices that measure parliamentary control (Karlas, 2012; Winzen, 2012). 
Overall, the articles focus on various parliamentary actors: committees, parliamentary parties and administrators. We argue that mainstreaming implies an increasing involvement of parliamentary actors beyond European affairs specialists. We should therefore also consider individual MPs when assessing the extent to which mainstreaming takes place inside national parliaments.

This brings us to the question: In what way would parliamentary actors become affected if we were to witness mainstreaming trends inside national parliaments? We propose that parliamentary actors are likely to be particularly affected by mainstreaming in four dimensions of parliamentary scrutiny. Since we understand the diffusion of responsibilities in EU affairs as a shift away from EACs, the first dimension concerns the growing number of sectoral committees and their members dealing with European affairs scrutiny, such as by issuing reports or opinions, and thereby describes 'decentralisation' processes. As this poses considerable strain on the administrative support system, the second dimension encompasses growing mainstreaming of EU affairs scrutiny at the administrative level. Our third dimension considers all parliamentary actors, namely administrators, parliamentary committees, political parties and other groups of MPs - for all of them we should witness increasing levels of inter-parliamentary cooperation and coordination. As a fourth dimension, we propose that mainstreaming can also be observed in the gradual increase in the number of plenary debates focusing on European issues because diffusion of responsibilities also means that more parliamentarians become involved in the scrutiny of EU affairs on a regular basis. Contrary to the other three dimensions, by which parliamentary scrutiny takes place behind closed doors, this one falls under open scrutiny of national parliaments in European affairs. On

\section{'... mainstreaming implies an increasing involvement of parliamentary actors beyond European affairs specialists.'}

the basis of our selection of existing research, we discuss early empirical manifestations of mainstreaming in the next section.

\section{MANIFESTATIONS OF THE MAINSTREAMING OF EU AFFAIRS SCRUTINY}

Although recent studies have not addressed our particular question as to whether we see an emerging trend towards mainstreaming of EU affairs within national parliaments, there is early evidence of mainstreaming affecting at least four main dimensions of parliamentary scrutiny, namely, the rising involvement of sectoral committees in EU affairs scrutiny; the adaptation of parliamentary staff to EU policymaking; increasing IPC beyond European affairs specialists; and the growing salience of EU affairs in plenary debates. In the following we present the results of our meta-analysis with respect to each of the four dimensions. It is important to note that these results are tentative, as we rely on a variety of studies that have applied various methods and that differ in their scope (see Table 1). Most of them actually do not provide quantifiable, and thus comparable, indicators with which we can assess developments for all twenty-seven or even twenty-eight national parliaments. This is why we complement our analysis with quantitative data from other first or secondary sources, as provided in Table 2 . In the last section we will propose ways about how to measure indicators of 
Table 1: Overview of research on the Europeanisation of national parliaments, post-Lisbon

\begin{tabular}{|c|c|c|c|c|}
\hline Study & Empirical focus & Method(s) & Countries studied & Time period \\
\hline $\begin{array}{l}\text { Auel et al } \\
(2015 a)\end{array}$ & $\begin{array}{l}\text { Plenary debates, EAC meetings, mandates } \\
\text { and resolutions, opinions within political } \\
\text { dialogue }\end{array}$ & Quantitative analysis of activities & EU-27 & $2010-2012$ \\
\hline $\begin{array}{l}\text { Auel and } \\
\text { Höing (2015) }\end{array}$ & $\begin{array}{l}\text { Crisis-related plenary debates, mandates } \\
\text { and resolutions, opinions within political } \\
\text { dialogue }\end{array}$ & Quantitative analysis of activities & EU-27 (lower houses) & $2010-2012$ \\
\hline $\begin{array}{l}\text { Auel and } \\
\text { Raunio } \\
\text { (2014) }\end{array}$ & Parliamentary debates & Quantitative content analysis & DE, FI, FR, GB (lower houses) & $\begin{array}{l}2002-2010 \\
2010-2012\end{array}$ \\
\hline $\begin{array}{l}\text { Closa and } \\
\text { Maatsch } \\
(2014)\end{array}$ & $\begin{array}{l}\text { Parliamentary approvals of the increased } \\
\text { budgetary capacity of the European } \\
\text { Financial Stability Facility }\end{array}$ & $\begin{array}{l}\text { Framing analysis of } \\
\text { parliamentary debates }\end{array}$ & $\begin{array}{l}\text { AT, BE, DE, EL, ES, FR, IE, LU, NL, } \\
\text { SI, SK (lower houses) }\end{array}$ & Autumn 2011 \\
\hline $\begin{array}{l}\text { Cooper } \\
(2015)\end{array}$ & $\begin{array}{l}\text { Inter-parliamentary coordination as } \\
\text { regards the first yellow card }\end{array}$ & $\begin{array}{l}\text { Process-tracing analysis and } \\
\text { expert interviews }\end{array}$ & $\begin{array}{l}\text { DK, FR (upper house), SE, GB } \\
\text { (lower house), PL (lower house), } \\
\text { FI, LU, PT, LT, MT, BE (lower house), } \\
\text { NL (lower house) }\end{array}$ & March-May 2012 \\
\hline $\begin{array}{l}\text { De Ruiter } \\
\text { (2013) }\end{array}$ & Committee and plenary debates & $\begin{array}{l}\text { Quantitative analysis of } \\
\text { scrutinising EU directives }\end{array}$ & GB and NL (lower houses) & $\begin{array}{l}\text { December } 2000- \\
\text { November } 2010\end{array}$ \\
\hline De Ruiter & Plenary debates & $\begin{array}{l}\text { Qualitative analysis of } \\
\text { scrutinising EU directives }\end{array}$ & GB and NL (upper houses) & December $2000-$ \\
\hline $\begin{array}{l}\text { De Wilde } \\
(2012)\end{array}$ & $\begin{array}{l}\text { Politicisation of EU budgets in plenary } \\
\text { debates and newspaper coverage }\end{array}$ & Claims-making analysis & DK, IE, NL (lower houses) & $\begin{array}{l}1993-1999 \\
2000-2006 \\
2007-2013\end{array}$ \\
\hline $\begin{array}{l}\text { Dörrenbächer } \\
\text { et al (2015) }\end{array}$ & $\begin{array}{l}\text { Parliamentary control over domestic } \\
\text { transposition of the Returns Directive }\end{array}$ & $\begin{array}{l}\text { Process-tracing analysis of } \\
\text { parliamentary documents }\end{array}$ & AT, DE, FR, NL & $2010-2012$ \\
\hline $\begin{array}{l}\text { Finke and } \\
\text { Herbel (2015) }\end{array}$ & $\begin{array}{l}\text { Political motivation of parliamentary } \\
\text { parties for committee referrals }\end{array}$ & Quantitative analysis & $\mathrm{DE}, \mathrm{FI}, \mathrm{FR}, \mathrm{GB}, \mathrm{IE}, \mathrm{IT}, \mathrm{PL}, \mathrm{SL}$ & $2000-2013$ \\
\hline $\begin{array}{l}\text { Gattermann } \\
\text { and Hefftler } \\
\text { (2015) }\end{array}$ & Participation in the EWS & $\begin{array}{l}\text { Quantitative analysis of number } \\
\text { of reasoned opinions }\end{array}$ & EU-27 & $2010-2013$ \\
\hline $\begin{array}{l}\text { Herranz- } \\
\text { Surrallés } \\
(2014)\end{array}$ & IPC in Foreign and Security Policy & $\begin{array}{l}\text { Parliamentary records, minutes } \\
\text { and position papers of two inter- } \\
\text { parliamentary meetings, and } \\
\text { interviews in } 2011\end{array}$ & EU-15 and EU 27 & 2001 and 2011 \\
\hline
\end{tabular}


Table 1: (Continued)

\begin{tabular}{|c|c|c|c|c|}
\hline Study & Empirical focus & Method(s) & Countries studied & Time period \\
\hline $\begin{array}{l}\text { Högenauer } \\
\text { and Neuhold } \\
\text { (2015) }\end{array}$ & $\begin{array}{l}\text { Role of parliamentary administrations in } \\
\text { EU affairs scrutiny }\end{array}$ & $\begin{array}{l}\text { Semi-structured interviews, } \\
\text { questionnaire with open } \\
\text { questions }\end{array}$ & $\mathrm{EU}-27$ & Post-Lisbon \\
\hline Huff (2015) & $\begin{array}{l}\text { National parliamentary scrutiny of the } \\
\text { EU's Common Foreign and Security Policy } \\
\text { and Common Security and Defence Policy }\end{array}$ & $\begin{array}{l}\text { Semi-structured interviews with } \\
\text { parliamentarians and staff }\end{array}$ & $\mathrm{DE}, \mathrm{DK}, \mathrm{FR}, \mathrm{GB}, \mathrm{IT}, \mathrm{NL}, \mathrm{PL}$ & $\begin{array}{l}\text { Post-Lisbon } \\
\text { (interview period: } \\
\text { March 2012-June } \\
\text { 2013) }\end{array}$ \\
\hline $\begin{array}{l}\text { Jensen and } \\
\text { Martinsen } \\
\text { (2015) }\end{array}$ & Executive scrutiny amid early agreements & $\begin{array}{l}\text { Survey addressed at EACs and } \\
\text { case studies (interviews, } \\
\text { secondary sources) }\end{array}$ & $\begin{array}{l}26 \text { EU countries (bar ES); DK, DE, } \\
\text { GB (case studies) }\end{array}$ & $\begin{array}{l}\text { Post-Lisbon } \\
\text { (survey/interview } \\
\text { period: } 2009- \\
\text { 2010) }\end{array}$ \\
\hline Karlas (2012) & $\begin{array}{l}\text { Four dimensions of control: scope, } \\
\text { decentralisation, influence mechanisms } \\
\text { and binding character }\end{array}$ & $\begin{array}{l}\text { Index-building from secondary } \\
\text { sources }\end{array}$ & $\mathrm{EU}-27$ & $2009-2010$ \\
\hline $\begin{array}{l}\text { Maatsch } \\
(2014)\end{array}$ & $\begin{array}{l}\text { Parliamentary party positions on anti- } \\
\text { crisis measures }\end{array}$ & $\begin{array}{l}\text { Discourse analysis of } \\
\text { parliamentary debates, QCA }\end{array}$ & $\begin{array}{l}\text { AT, BE, DE, EL, ES, FR, IE, SI (lower } \\
\text { houses) }\end{array}$ & July-October 2011 \\
\hline $\begin{array}{l}\text { Neuhold and } \\
\text { Högenauer } \\
\text { (forthcoming) }\end{array}$ & Parliamentary administrations and IPC & Semi-structured interviews & $\mathrm{EU}-27$ & Post-Lisbon \\
\hline $\begin{array}{l}\text { Peters et al } \\
\text { (2014) }\end{array}$ & $\begin{array}{l}\text { Parliamentary involvement in the } \\
\text { decision-making process of the EU's anti- } \\
\text { piracy mission Atalanta including } \\
\text { information, monitoring and IPC }\end{array}$ & $\begin{array}{l}\text { In-depth case study, background } \\
\text { interviews and analysis of } \\
\text { parliamentary documents }\end{array}$ & $\begin{array}{l}\text { BE, DE, ES, NL (focus on lower } \\
\text { houses) }\end{array}$ & $2008-2013$ \\
\hline $\begin{array}{l}\text { Puntscher } \\
\text { Riekmann and } \\
\text { Wydra (2013) }\end{array}$ & Plenary debates on crisis-related topics & Qualitative content analysis & AT, DE, IT (lower houses) & $2010-2012$ \\
\hline $\begin{array}{l}\text { Strelkov } \\
\text { (2015) }\end{array}$ & $\begin{array}{l}\text { Interaction of parliamentary party groups, } \\
\text { administrators and committees }\end{array}$ & Semi-structured interviews & $\mathrm{CZ}, \mathrm{RO}, \mathrm{SE}$ & $\begin{array}{l}\text { Post-Lisbon } \\
\text { (interview period: } \\
\text { October 2010- } \\
\text { November 2013) }\end{array}$ \\
\hline $\begin{array}{l}\text { Winzen } \\
(2012)\end{array}$ & $\begin{array}{l}\text { Three indicators of control: information, } \\
\text { processing and enforcement }\end{array}$ & $\begin{array}{l}\text { Index-building from secondary } \\
\text { sources }\end{array}$ & $\mathrm{EU}-6$ to $\mathrm{EU}-27$ & $1958-2010$ \\
\hline
\end{tabular}


Table 2: Early evidence of the mainstreaming of EU affairs inside national parliaments

\begin{tabular}{|c|c|c|c|c|c|c|c|c|c|}
\hline \multirow{2}{*}{$\begin{array}{l}\text { Dimension } \\
\text { Indicator }\end{array}$} & & \multicolumn{3}{|c|}{ Sectoral committees } & \multirow{3}{*}{$\begin{array}{l}\text { Administration } \\
\text { Staff support for } \\
\text { sectoral } \\
\text { committees }^{c}\end{array}$} & \multicolumn{2}{|c|}{ IPC } & \multicolumn{2}{|c|}{ Debates } \\
\hline & & $\begin{array}{c}\text { Involvement } \\
\text { in EU } \\
\text { scrutiny }^{a}\end{array}$ & $\begin{array}{c}\text { Involvement in } \\
\text { drafting reasoned } \\
\text { opinions }\end{array}$ & $\begin{array}{c}\text { Recent } \\
\text { strengthening of } \\
\text { sectoral } \\
\text { committees }^{c}\end{array}$ & & $\begin{array}{l}\text { Involvement } \\
\text { by sectoral } \\
\text { committees }^{d}\end{array}$ & $\begin{array}{l}\text { Liaison } \\
\text { officers in } \\
\text { Brussels }^{\mathrm{e}}\end{array}$ & $\begin{array}{l}\text { Reasoned } \\
\text { opinions } \\
\text { adopted by } \\
\text { plenary }\end{array}$ & $\begin{array}{l}\text { Use of } \\
\text { EU } \\
\text { debates }^{f}\end{array}$ \\
\hline \multicolumn{9}{|l|}{ Country } & \\
\hline \multirow[t]{2}{*}{ Austria } & U & & No & $\mathrm{N} / \mathrm{A}$ & $\mathrm{N} / \mathrm{A}$ & Fully & Joint & No & Medium \\
\hline & $\mathrm{L}$ & No & No & $\mathrm{N} / \mathrm{A}$ & $\mathrm{N} / \mathrm{A}$ & Highly & Joint & No & Medium \\
\hline \multirow[t]{2}{*}{ Belgium } & U & & Fully & No change & EAC staff & Highly & Yes & Always & Low \\
\hline & $\mathrm{L}$ & Occasionally & No, adopting only & No change & EAC staff & Highly & Yes & Sometimes & Low \\
\hline Bulgaria & & Regularly & Consulted by EAC & Since accession & EAC staff, CU & Highly & Yes & No & Low \\
\hline Cyprus & & Occasionally & No & $\mathrm{N} / \mathrm{A}$ & $\mathrm{N} / \mathrm{A}$ & Highly & Yes & No & Low \\
\hline \multirow{2}{*}{$\begin{array}{l}\text { Czech } \\
\text { Republic }\end{array}$} & $U$ & & No & Since accession & EAC staff, CU & Often & Yes & Always & High \\
\hline & $\mathrm{L}$ & Occasionally & Consulted by EAC & Since accession & EAC staff, CU & Highly & Yes & Sometimes & Low \\
\hline Denmark & & Occasionally & Highly & 1994, 2011 & CU, EU advisors & Highly & Yes & No & Low \\
\hline Estonia & & Regularly & No & $\mathrm{N} / \mathrm{A}$ & N/A & Highly & Yes & Always & Low \\
\hline Finland & & Regularly & No & $\mathrm{N} / \mathrm{A}$ & $\mathrm{N} / \mathrm{A}$ & Fully & Yes & Always & Medium \\
\hline \multirow[t]{2}{*}{ France } & $\mathrm{U}$ & & No, adopting only & 2008 & EAC staff & Highly & No & Sometimes & Low \\
\hline & $\mathrm{L}$ & Regularly & No, adopting only & 2008 & EAC staff & Often & Yes & Sometimes & Low \\
\hline \multirow[t]{2}{*}{ Germany } & $\bar{U}$ & & Fully & Incrementally & Regional ministries & Highly & Yes & Usually & Low \\
\hline & $\mathrm{L}$ & Regularly & Fully & Incrementally & $\mathrm{CU}$ & Highly & Yes & Usually & Medium \\
\hline Greece & & Occasionally & Jointly with EAC & NI & NI & Highly & Yes & No & Low \\
\hline Hungary & & Regularly & No & & & Highly & Yes & Always & Low \\
\hline \multirow[t]{2}{*}{ Ireland } & $U$ & & Consulted by EAC & 2011 & Own EU staff, CU & Fully & Joint & Always & Low \\
\hline & $\mathrm{L}$ & Occasionally & Consulted by EAC & 2011 & Own EU staff, CU & Highly & Joint & Always & Medium \\
\hline \multirow[t]{2}{*}{ Italy } & U & & Jointly with EAC & 2006 & $\mathrm{CU}$ & Highly & Yes & Sometimes & Medium \\
\hline & $\mathrm{L}$ & Regularly & No & NI & $\mathrm{CU}$ & Fully & Yes & Sometimes & Low \\
\hline Latvia & & Occasionally & No & $\mathrm{N} / \mathrm{A}$ & $\mathrm{N} / \mathrm{A}$ & Highly & Yes & No & Low \\
\hline Lithuania & & Regularly & Consulted by EAC & Since EWS & None & Highly & Yes & Always & Low \\
\hline Luxembourg & & Regularly & Fully & 2005 & $\mathrm{CU}$ & Highly & Yes & Usually & Low \\
\hline Malta & & No & No & $\mathrm{N} / \mathrm{A}$ & $\mathrm{N} / \mathrm{A}$ & Highly & Yes & $\mathrm{NI}$ & Low \\
\hline $\begin{array}{l}\text { The } \\
\text { Netherlands }\end{array}$ & $\mathrm{U}$ & & Fully & $2005-2006$ & None & Fully & Joint & Always & Low \\
\hline
\end{tabular}


Table 2: (Continued)

\begin{tabular}{|c|c|c|c|c|c|c|c|c|c|}
\hline \multirow{2}{*}{$\frac{\text { Dimension }}{\text { Indicator }}$} & \multicolumn{4}{|c|}{ Sectoral committees } & \multirow{3}{*}{$\begin{array}{l}\text { Administration } \\
\text { Staff support for } \\
\text { sectoral } \\
\text { committees }^{c}\end{array}$} & \multicolumn{2}{|c|}{ IPC } & \multicolumn{2}{|c|}{ Debates } \\
\hline & & $\begin{array}{l}\text { Involvement } \\
\text { in EU } \\
\text { scrutiny }\end{array}$ & $\begin{array}{l}\text { Involvement in } \\
\text { drafting reasoned } \\
\text { opinions }^{\mathrm{b}}\end{array}$ & $\begin{array}{c}\text { Recent } \\
\text { strengthening of } \\
\text { sectoral } \\
\text { committees }^{c}\end{array}$ & & $\begin{array}{l}\text { Involvement } \\
\text { by sectoral } \\
\text { committees }^{d}\end{array}$ & $\begin{array}{l}\text { Liaison } \\
\text { officers in } \\
\text { Brussels }^{\mathrm{e}}\end{array}$ & $\begin{array}{l}\text { Reasoned } \\
\text { opinions } \\
\text { adopted by } \\
\text { plenary }^{\mathrm{b}}\end{array}$ & $\begin{array}{l}\text { Use of } \\
\text { EU } \\
\text { debates }^{f}\end{array}$ \\
\hline \multicolumn{9}{|l|}{ Country } & \\
\hline \multirow{3}{*}{ Poland } & $\mathrm{L}$ & Regularly & Consulted by EAC & 2006 & Own EU staff & Highly & Joint & Always & Medium \\
\hline & $\mathrm{U}$ & & Jointly with EAC & Since EWS & $\mathrm{CU}$ & Highly & Yes & Always & Low \\
\hline & $\mathrm{L}$ & No & No & $\mathrm{N} / \mathrm{A}$ & N/A & Highly & Yes & Always & Low \\
\hline Portugal & & Occasionally & Consulted by EAC & 2010,2013 & None & Highly & Yes & Usuailly & Low \\
\hline \multirow[t]{2}{*}{ Romania } & $\mathrm{U}$ & & Highly & Since accession & $\mathrm{NI}$ & Fully & No & NI & Low \\
\hline & $\mathrm{L}$ & No & Highly & Since accession & $\mathrm{CU}$ & Highly & Yes & NI & Low \\
\hline Slovakia & & Occasionally & Consulted by EAC & N/A & $\mathrm{N} / \mathrm{A}$ & Highly & Yes & NI & Low \\
\hline \multirow{2}{*}{ Slovenia } & $\mathrm{U}$ & & Consulted by EAC & 2010 & NI & $\mathrm{NI}$ & No & Always & Low \\
\hline & $\mathrm{L}$ & Regularly & Fully & 2010 & NI & Highly & Yes & Sometimes & Low \\
\hline \multirow{2}{*}{ Spain } & $U$ & & No & N/A & $\mathrm{N} / \mathrm{A}$ & Fully & Yes & Sometimes & Low \\
\hline & $\mathrm{L}$ & Occasionally & No & N/A & $N / A$ & Highly & Yes & Sometimes & Low \\
\hline \multirow{3}{*}{$\begin{array}{l}\text { Sweden } \\
\text { UK }\end{array}$} & & Occasionally & Fully & 1997,2007 & CU, own EU staff & Fully & Yes & Always & Medium \\
\hline & $U$ & & No & N/A & $\mathrm{N} / \mathrm{A}$ & Seldom & Yes & Always & Medium \\
\hline & $\mathrm{L}$ & Occasionally & No & N/A & N/A & Highly & Yes & Always & Low \\
\hline
\end{tabular}

${ }^{a}$ On the basis of Karlas (2012); upper houses were not assessed on this indicator.

${ }^{\mathrm{b}}$ On the basis of a report of the Legislative Dialogue Unit of the European Parliament/Directorate for Relations with National Parliaments (2013); 'highly' involved means that the draft may be finalised by the EAC.

'On the basis of interviews and Hefftler et al (2015); only compiled for those chambers where sectoral committees play a role.

${ }^{\mathrm{d}}$ On the basis of percentage of MPs from sectoral committees taking part in Brussels committee meetings, 2009-2012 (Gattermann, 2013); fully involved $=100$ per cent, highly $=61-99$ per cent, often $=31-60$ per cent, seldom $=0-30$ per cent.

eOn the basis of Neuhold and Högenauer (forthcoming).

fOn the basis of the score for debates by Auel et al (2015b). Low = activity score from 0 to 0.29 ; medium $=0.3-0.59$; high $\leq 0.6$; the score takes into account the number and duration of plenary debates on EU issues, as well as the relative time spent debating EU issues.

Abbreviations: $\mathrm{L}=$ lower house, $\mathrm{U}=$ upper house, $\mathrm{CU}=\mathrm{a}$ central unit that serves all committees (e.g., a legal, research or information service), $\mathrm{NI}=$ no information 
mainstreaming more systematically in future research.

As regards our first dimension, few existing studies have investigated the extent to which sectoral committees become increasingly involved in EU affairs at the expense of EACs over time. One exception is the study by Winzen (2012: 667), which shows that sectoral committees in the EU-9 member states have already become more and more and active in EU affairs scrutiny between 1973 and 2009. Others provide crosssectional snapshots of European affairs scrutiny for the time period after Lisbon. In this respect, Jensen and Martinsen (2015) analyse, among other things, the scrutiny of early agreements between the Council of Ministers and the European Parliament by sectoral committees. They show that sectoral committees are sometimes involved in the Danish Folketing and the British House of Commons, while the specialised EU subcommittees in the House of Lords as well as sectoral committees in both German chambers are highly involved in the scrutiny of early agreements.

Karlas (2012) provides a more comprehensive overview of the extent to which EU affairs scrutiny has been decentralised by means of involving sectoral committees after Lisbon. His results for lower houses are reported in Table 2, column 'Involvement in EU scrutiny'. Generally, only four out of twenty-seven chambers, namely, the Austrian Nationalrat, the Polish Sejm, the Romanian Camera as well as the Maltese Parliament have kept EU affairs scrutiny within the jurisdiction of EACs; twelve chambers occasionally involve sectoral committees, while the remaining eleven chambers do so on a regular basis. These findings also correspond with parliamentary scrutiny in specific policy areas. Huff (2015: 404), for instance, finds by expert interviews that the respective Foreign and Defence Committees of the Dutch Tweede Kamer and the Italian Camera are particularly active in the scrutiny of the EU's Common Foreign and Security Policy and Common Security and Defence Policy, while the respective committees in the Polish Sejm and Danish Folketing hardly become involved.

Another striking example is the process of drafting reasoned opinions as part of EWS. As shown in Table 2 (column 'Involvement in drafting reasoned opinions'), almost two-thirds of all parliaments involve sectoral committees to at least some extent in the formulation of reasoned opinions. Some of them, among them the German, Swedish and Luxembourgish parliaments even delegate this task entirely to sectoral committees. While we are unable to measure any trend over time, this indicator shows that some national parliaments have particularly responded to the new provisions of the Lisbon Treaty by involving their sectoral committees more actively. The column 'Recent strengthening of sectoral committees' in Table 2 shows that some have strengthened their sectoral committees after Lisbon, others even before the new treaty came into force, perhaps in anticipation of the new political and legal developments.

Regarding our second dimension, parliamentary practice suggests that the administrative support system plays an important advisory function in most European parliaments today (Högenauer and Neuhold, 2015; van Keulen, 2012). As sectoral committees now increasingly require advice on EU policies and procedures, the result has been growing mainstreaming of EU affairs scrutiny at the administrative level. It can take a variety of forms, including specialised EU staff among all committee staff (as in the case of the Dutch Tweede Kamer or the Swedish Riksdag) or of a sufficiently large common EU unit with staff who specialise in different EU policy areas (e.g., the Danish Folketing, the German Bundestag or the 
Luxemburgish Chamber of Representatives) (Högenauer and Christiansen, 2015). Less resource-intensive options for mainstreaming include a simple transfer of EU support tasks to 'ordinary' committee staff who will have to accept that European issues are part of the work of their sectoral committees (e.g., the Dutch Eerste Kamer, Högenauer, 2015), or putting existing EAC staff in charge of advising sectoral committees on EU affairs (e.g., the Belgian Senate, cf. Högenauer and Neuhold, 2015). As the column entitled 'Staff support for sectoral committees' in Table 2 shows, even among those parliaments that have delegated the competence for EU affairs scrutiny wholly, or in part, to sectoral committees, there are still many cases in which only the EAC has EU experts at its disposal. Mainstreaming has thus progressed less far in the second dimension compared with the first dimension.

Our third dimension - IPC - has been particularly encouraged by the Lisbon Treaty and other new legal provisions. Before Lisbon, COSAC (Conference of Parliamentary Committees for Union Affairs of Parliaments of the European Union) played a central role. Over recent years, new, informal and formalised forms of IPC have been established between parliamentary committees, political parties and other groups of MPs (for an overview, see Hefftler and Gattermann, 2015). Although Herranz-Surrallés (2014: 958) argues that inter-parliamentary relations in the field of foreign and security policy 'have become less structured and more strained', Peters et al (2014) find that MPs appreciated formal as well as informal ways of IPC for their networking opportunities and information exchange regarding the EU's anti-piracy mission Atalanta. Moreover, there is evidence that mainstreaming of IPC takes place at the general level of inter-parliamentary exchange. As shown in Table 2 (column 'Involvement by sectoral committees'),
Gattermann (2013) observes increased participation of non-EU specialists from national parliaments, since generally more MPs from sectoral committees, rather than EAC members, have participated in inter-parliamentary committee meetings in Brussels between 2009 and 2012. Only members of sectoral committees from the British House of Lords are seldom involved, which might be explained by the fact that the respective EAC consists of several specialised EU sub-committees. Moreover, Table 2 (column 'Liaison officers in Brussels') shows that most national parliaments also cooperate on the administrative level in the post-Lisbon era. Neuhold and Högenauer (forthcoming) find that the number of liaison officers in Brussels has steadily increased since 1991, when the Danish Folketing installed the first officer in Brussels. According to them, liaison officers are particularly important for the information exchange between parliaments. The network of national parliament representatives in Brussels, for instance, also played a major role in the coordination of the first yellow card within the EWS (Cooper, 2015), which suggests that they continue to be an indicator for mainstreaming in the dimension of IPC in the future. Thus far, however, we find little evidence for IPC at the party level postLisbon, although research has identified them as key players in EU affairs scrutiny (Finke and Herbel, 2015; Strelkov, 2015) and acknowledges the importance of transnational party networks in the case of Atalanta (Peters et al, 2014: 443).

Our last dimension concerns the growing salience of European affairs in plenary debates. Bergmann et al (2003: 175) show that before the Nice Treaty came into force in 2003, plenary meetings were hardly used for European affairs scrutiny in the EU-15. However, we expect the Lisbon Treaty to have triggered a shift in responsibilities over European affairs in national parliaments. For instance, 
following the introduction of the EWS, we find that in most cases the plenary formally adopts reasoned opinions as shown in the column 'Reasoned opinions adopted by plenary' in Table 2. This also implies that debates precede the adoption by the plenary. This is particularly the case for the French and Polish Senate as well as in both chambers of the Spanish Parliament (Legislative Dialogue Unit of the European Parliament/Directorate for Relations with National Parliaments, 2013). However, even though the plenaries of sixteen chambers are 'always' responsible for the adoption of reasoned opinions, the low use of this instrument in practice does not necessarily infer frequent debates of EU legislative proposals, except perhaps for the most active chambers in the EWS, including the Swedish Riksdag, the French Senate and the Dutch Tweede Kamer (Gattermann and Hefftler, 2015).

Plenary debates take place in public with direct access by the media and citizens, which means that both mainstreaming processes and the parliamentary scrutiny of EU affairs are likely to become more visible to the public. Yet, the last column of Table 2 shows that there is considerable variation among parliaments: only a few make very active use of debates, but there are still many in which EU affairs are sidelined. These data are provided by Auel et al (2015b), on which Auel and her co-authors in the three above-cited studies all rely (see Table 1). By contrast, in his two-country comparison between 2000 and 2010, De Ruiter $(2013,2015)$ shows that there are numerically more references to EU directives in plenary debates in the United Kingdom than in the Netherlands. This is also reflected by the use of debates in both upper houses by the evidence presented in Table 2. However, the data of Auel et al (2015b) suggest that EU affairs are less frequently debated in the House of Commons than in the Dutch Tweede Kamer.
Furthermore, we also find other kinds of indicators for a potential mainstreaming trend in the qualitative analyses of parliamentary debates and party positions. These can be generally understood as increasing awareness for EU-level decisions, including financial and fiscal issues (Puntscher Riekmann and Wydra, 2013), or EU migration law (Dörrenbächer et $a l, 2015)$. In particular, De Wilde (2012) contends that politicisation over the EU budget has the potential to alleviate the 'constraining dissensus' in national parliaments; and Maatsch (2014) shows that the Eurozone crisis has led some parliamentary parties in debtor countries to alter their political ideology regarding their positions towards anti-crisis measures.

Taken together, the early evidence suggests a greater involvement of committees, support staff, political parties and ultimately MPs in European affairs. However, the extent of mainstreaming varies greatly both across parliaments and across dimensions. Table 2 illustrates that there is no one-size-fits-all approach to mainstreaming. This poses a challenge for researchers - and especially comparativists, as these diverse approaches ought to be factored into attempts to measure 'level of activity' or 'strength' across parliaments.

\section{A NEW TREND IN EUROPEANISATION - NEW CHALLENGES FOR RESEARCH?}

European integration has reached a point where the participation of national parliaments in EU affairs is not solely motivated by the growing transfer of competences to the European level. The Treaty of Lisbon has created new opportunities; the sovereign debt crisis has made at least some aspects of EU affairs politically and electorally salient. However, 
'... the early evidence suggests a greater involvement of committees, support staff, political parties and ultimately MPs in European affairs.'

in line with historical institutionalism, we assume that national parliaments embrace mainstreaming depending on the presence of a number of intervening factors: time and resource costs, the political salience of European politics in that country and perceived success of the existing system. There is variation between parliaments in the extent of mainstreaming and in the timing of mainstreaming. The Dutch parliament, for example, has made extensive reforms towards mainstreaming in 2006 (Högenauer, 2015), whereas the Portuguese Assembleia da República only adopted moderate reforms in May 2012, by which the EAC now explicitly shares competences in the scrutiny of European affairs with the plenary and other committees. $^{3}$

Overall, the trend towards mainstreaming not only affects parliaments themselves, but also how research into the Europeanisation of parliaments and their behaviour in EU affairs scrutiny has to be designed. Comparativists are particularly affected, as one can no longer compare the powers and levels of activities of EACs: tasks that are being performed by EACs in one parliament may be performed by sectoral committees in other parliaments. However, in order to factor in mainstreaming in comparative studies of parliaments, more systematic approaches towards the extent of mainstreaming across parliaments in different elements of parliamentary scrutiny are needed. Thus, how can we measure mainstreaming within our four dimensions?
As regards 'decentralisation' towards sectoral committees, one can begin by comparing the formal powers of these committees as specified in the rules of procedure. In addition, in order to measure their active involvement in practice, Raunio (2009: 326) suggests estimating the share of committee time spent on EU legislation to assess the degree of Europeanisation. Another study should investigate the number of EU issues on the agenda of sectoral committees, alongside the resources invested in scrutinising them (e.g., including invitations to government representatives and European politicians to report before the committee or hearings with experts). A closer look at their composition would also answer questions about whether a new group of cross-issue EU specialists is emerging. This could be accomplished by tracing the relationship between individual MPs' career paths and legislative behaviour.

To measure the extent of mainstreaming in IPC, future research should trace the developments individually for each parliament over time. This provides information about who attends these meetings, how often and why. Ultimately, such research would determine whether (initial) non-EU specialists become involved more often in IPC, or whether the same faces show up every time, rendering European affairs accessible only to a few experts in national parliaments. Surveys would furthermore give insights about attendance at informal meetings and the individual motives of MPs to take part in IPC (see also Raunio, 2000; Miklin and Crum, 2011). Surveys and interviews could also provide answers regarding the mainstreaming of EU staff and assess whether and to what extent staff are specialised in specific policy areas. The extent to which mainstreaming affects the administrative level can also be studied by looking at the organisation or number of staff. In particular, one can assess whether sectoral committees have their 
own EU experts attached to them, or whether the main EU support unit contains staff that is explicitly in charge of supporting sectoral committees.

Lastly, a content analysis offers a key opportunity for the study of parliamentary debates enabling the assessment of cross-country, cross-issue and inter-temporal variation. A quantitative study could, for instance, investigate to what extent plenary debates deal with European affairs by proportional measures, and thereby assess the salience and visibility of EU issues (e.g., De Wilde, 2011); and which parliamentary actors become publicly active in European affairs, such as by asking parliamentary questions (e.g., see De Ruiter, 2014) to enquire whether we witness an increase in EU specialists across policy areas. A qualitative analysis would answer questions about the framing of European issues or the tone of parliamentary actors towards the EU in debates (e.g., see Closa and Maatsch, 2014).

It is likely that the intensities to which mainstreaming in each of our four dimensions occurs are to some extent interlinked with each other. Even if they have formal powers, sectoral committees are less likely to become actively involved in EU affairs scrutiny if they are unable to draw on sufficient resources, that is, support staff. As Högenauer and Christiansen (2015) show, not all parliaments have increased their staff following Lisbon. Similarly, in parliaments where the EAC has a strong institutionalised position, undermining the role of sectoral committees in European affairs scrutiny, the chances are lower that European affairs are frequently debated in plenary. Most EACs are granted the right to debate and vote on European issues on behalf of the whole parliament (see Raunio 2009: 319). Thus, in parliaments where the EAC remains the central body for European affairs scrutiny, the likelihood of the plenary debating EU issues diminishes. As

\section{'It is likely that the intensities to which mainstreaming in each of our four dimensions occurs are to some extent interlinked with each other.'}

shown in Table 2, EU issues are prominent on the plenary agenda of, among others, the German Bundestag, whose sectoral committees have had an influence in EU affairs for a long time. Conversely, in the Slovakian National Council, where the EAC has a central status, EU issues are less frequently debated in the open chamber.

Moreover, there are examples in which mainstreaming has occurred only in certain areas. As Table 2 demonstrates, most MPs of the two Austrian chambers attending inter-parliamentary committee meetings are from sectoral committees, despite the fact that - internally - EU affairs are still dealt with by the EAC. Conversely, the Belgian Senate has decentralised EU affairs scrutiny to the sectoral committees, but has too few EU staff to allow for specialisation on policy sectors (Högenauer and Neuhold, 2015). In sum, we argue that mainstreaming has varying characteristics, which makes a better understanding of the transformations more urgent.

Overall, the effects of these changes on the functioning of parliaments are fairly extensive and change how the Europeanisation of parliaments and their scrutiny of EU affairs should be studied by academics. At the same time, the number of mainstreaming dimensions proposed in this article is not finite. There are activities outside the immediate parliamentary arena (and related to the communication function of parliaments and their members) that could also be affected by 
mainstreaming: most notably parliamentary election campaigns and constituency services of individual MPs. Parliamentarians who do not invest resources in becoming more involved in European affairs by means of their committee membership, via IPC or by contributions in plenary debates, are unlikely to fight electoral campaigns over European integration or exchange their views directly with their constituents. Conversely, those who are EU specialists in their national parliaments are constrained in their communication function if mainstreaming does not take place across parties and committees or in plenary debates.

Hence, future research needs to take into account the changing organisation of EU affairs scrutiny, both in the formulation of research questions, the choice of study design and the application of appropriate methods to study mainstreaming of EU affairs in national parliaments. In addition, as the findings of Miklin (2014) illustrate, researchers should be open to the possible wider effects of mainstreaming in party behaviour and electoral politics.

\section{Acknowledgements}

The authors wish to thank their colleagues from the former OPAL (Observatory of Parliaments after Lisbon) project, Davor Jancic, Mads Dagnis Jensen, the anonymous reviewers and the editor for helpful comments and suggestions. Authors are listed alphabetically. The usual disclaimer applies.

This work was conducted during the OPAL project and jointly funded by the DFG [WE 954/13-1], the NWO [464-10074] and the ESRC [ES/I014853/1] as part of an Open Research Area Fund (ARN-DFG-ESRC-NWO).

\section{Notes}

1 As captured, for example, by Ladrech's (1994) definition of Europeanisation as a top-down process whereby European 'political and economic dynamics become part of the organizational logic of national politics and policy-making' (69).

2 Winzen (2012: 663-65) noted, however, that the pre-2004 member states generally retained weaker scrutiny systems relative to those of new member states.

3 Law no. 43/2006 of 25 August 2006, as amended by Law no. 21/2012 of 17 May 2012 (see http://www .en.parlamento.pt/Legislation/Law21_2012EN.pdf, last accessed 18 September 2012), Article 6.1.

\section{References}

Armstrong, K.A. and Bulmer, S. (1998) The Governance of the Single European Market, Manchester: Manchester University Press.

Auel, K. (2005) 'Introduction: The Europeanization of parliamentary democracy', Journal of Legislative Studies 11(3-4): 303-18.

Auel, K. and Benz, A. (2005) 'The politics of adaptation: Europeanisation of national parliamentary systems', Journal of Legislative Studies 11(3-4): 372-93.

Auel, K. and Höing, O. (2015) 'National parliaments and the Eurozone crisis: Taking ownership in difficult times?' West European Politics 38(2): 375-395.

Auel, K. and Raunio, T. (2014) 'Debating the state of the union? Comparing parliamentary debates on EU issues in Finland, France, Germany and the United Kingdom', Journal of Legislative Studies 20(1): 13-28.

Auel, K., Rozenberg, O. and Tacea, A. (2015a) 'To scrutinise or not to scrutinise? Explaining variation in EU-related activities in national parliaments', West European Politics 38(2): 282-304. 
Auel, K., Rozenberg, O. and Tacea, A. (2015b) 'Fighting Back? And if Yes, How? Measuring Parliamentary Strength and Activity in EU Affairs', in C. Hefftler, C. Neuhold, O. Rozenberg and J. Smith (eds.) The Palgrave Handbook of National Parliaments and the European Union, London: Palgrave Macmillan, pp. 60-93.

Bergman, T., Müller, W.C., Strøm, K. and Blomgren, M. (2003) 'Democratic Delegation and Accountability: Cross-National Patterns', in K. Strøm, W.C. Müller and T. Bergman (eds.) Delegation and Accountability in Parliamentary Democracies, Oxford: Oxford University Press, pp. 109-220.

Carter, C. and McLeod, A. (2006) 'The Scottish Parliament and the European Union: Analysing Regional Parliamentary Engagement', in S. Weatherill and U. Bernitz (eds.) The Role of Regions and Subnational Actors in Europe, Oxford; Portland: Hart Publishing, pp. 67-88.

Closa, C. and Maatsch, A. (2014) 'In a spirit of solidarity? Justifying the European financial stability facility (EFSF) in national parliamentary debates', Journal of Common Market Studies 52(4): 26-842.

Cooper, I. (2015) 'A yellow card for the striker: National parliaments and the defeat of EU legislation on the right to strike', Journal of European Public Policy 22(10): 1406-1425.

De Ruiter, R. (2013) 'Under the radar? National parliaments and the ordinary legislative procedure in the European Union', Journal of European Public Policy 20(8): 1196-1212.

De Ruiter, R. (2014) 'Public parliamentary activities and open methods of coordination', Journal of Legislative Studies 20(1): 62-77.

De Ruiter, R. (2015) 'Houses of abstention or houses of reflection? Upper houses in EU member states and the ex ante scrutiny of EU legislation', Journal of European Integration 37(3): 391-407.

De Wilde, P. (2011) 'Ex ante vs. ex post: The trade-off between partisan conflict and visibility in debating EU policy-formulation in national parliaments', Journal of European Public Policy 18(5): 72-689.

De Wilde, P. (2012) 'Politicisation of the EU budget: Conflict and the constraining dissensus', West European Politics 35(5): 1075-94.

Dimitrakopoulos, D.G. (2001) 'Incrementalism and path dependence: European integration and institutional change in national parliaments', Journal of Common Market Studies 39(3): 405-22.

Dörrenbächer, N., Mastenbroek, E. and Toshkov, D.D. (2015) 'National parliaments and transposition of EU law: A matter of coalition conflict?' Journal of Common Market Studies 53(5): 1010-1026.

European Scrutiny Committee, House of Commons. (2013) '24th Report of Session 2013-14: Reforming the European Scrutiny System in the House of Commons', vols. I-III. London: The Stationary Office.

Exadaktylos, T. and Radaelli, C.M. (2009) 'Research design in European studies: The case of Europeanization', Journal of Common Market Studies 47(3): 507-30.

Finke, D. and Herbel, A. (2015) 'Beyond rules and resources: Parliamentary scrutiny of EU policy proposals', European Union Politics. doi:10.1177/1465116515584202 Published online before print 27 May 2015; pages 1-24.

Gattermann, K. (2013) 'Brussels Calling!? National Parliamentarians' Participation in Inter-Parliamentary Committee Meetings'. EUSA Biennial Conference, Baltimore.

Gattermann, K. and Hefftler, C. (2015) 'Beyond institutional capacity: Political motivation and parliamentary behaviour in the early warning system', West European Politics 38(2): 305-34.

Hall, P.A. and Taylor, R.C.R. (1996) 'Political science and the three new institutionalisms', Political Studies 44(5): 936-57.

Hefftler, C. and Gattermann, K. (2015) 'Interparliamentary Cooperation in the European Union: Patterns, Problems and Potential', in C. Hefftler, C. Neuhold, O. Rozenberg and J. Smith (eds.) The Palgrave Handbook of National Parliaments and the European Union, London: Palgrave Macmillan, pp. 94-115.

Hefftler, C., Neuhold, C., Rozenberg, O. and Smith, J. (eds.) (2015) The Palgrave Handbook of National Parliaments and the European Union, London: Palgrave Macmillan.

Herranz-Surrallés, A. (2014) 'The EU's multilevel parliamentary (battle)field: Inter-parliamentary cooperation and conflict in foreign and security policy', West European Politics 37(5): 957-75.

Högenauer, A.L. (2015) 'The Dutch Parliament and EU Affairs: Decentralizing Scrutiny', in C. Hefftler, C. Neuhold, O. Rozenberg and J. Smith (eds.) The Palgrave Handbook of National Parliaments and the European Union, London: Palgrave Macmillan, pp. 252-71.

Högenauer, A.L. and Christiansen, T. (2015) 'Parliamentary Administrations in the Scrutiny of EU Decision-Making', in C. Hefftler, C. Neuhold, O. Rozenberg and J. Smith (eds.) The Palgrave Handbook of National Parliaments and the European Union, London: Palgrave Macmillan, pp. 116-32.

Högenauer, A.L. and Neuhold, C. (2015) 'National parliaments after Lisbon: Administrations on the rise?' West European Politics 38(2): 255-354.

Huff, A. (2015) 'Executive privilege reaffirmed? Parliamentary scrutiny of the CFSP and CSDP', West European Politics 38(2): 396-415. 
Jensen, M.D. and Martinsen, D. (2015) 'Out of time? National parliaments and early decision-making in the European Union', Government and Opposition 50(2): 40-270.

Karlas, J. (2012) 'National parliamentary control of EU affairs: Institutional design after enlargement', West European Politics 35(5): 1095-113.

Ladrech, R. (1994) 'Europeanization of domestic politics and institutions: The case of France', Journal of Common Market Studies 32(1): 69-88.

Legislative Dialogue Unit of the European Parliament/Directorate for Relations with National Parliaments. (2013) 'National parliaments' internal procedures for subsidiarity checks', http://www.europarl. europa.eu/webnp/webdav/site/myjahiasite/shared/Publications/subsidiarity\%20procedures/Table\% 20internal\%20procedures\%20for\%20subsidiarity\%20check\%2008.04.13.pdf (last updated 8 April 2013; accessed 13 August 2015).

Maatsch, A. (2014) 'Are we all Austerians now? An analysis of national parliamentary parties' positioning on anti-crisis measures in the Eurozone', Journal of European Public Policy 21(1): 96-115.

Machill, M., Beiler, M. and Fischer, C. (2006) 'Europe-topics in Europe's media: The debate about the European public sphere: A meta-analysis of media content analyses', European Journal of Communication 21(1): 57-88.

Maurer, A. and Wessels, W. (eds.) (2001) National Parliaments on their Ways to Europe: Losers or Latecomers?, Baden-Baden: Nomos.

Miklin, E. (2014) 'From "sleeping giant" to left-right politicization? National party competition on the EU and the euro crisis', Journal of Common Market Studies 52(6): 1199-1206.

Miklin, E. and Crum, B. (2011) 'Inter-Parliamentary Contacts of Members of the European Parliament. Report of a Survey', RECON Online Working Paper 2011/08, Oslo: ARENA - Centre for European Studies.

Neuhold, C. and Högenauer, A.L. (forthcoming) 'An information network of officials? Dissecting the role and nature of the network of parliamentary representatives in the EP', Journal of Legislative Studies.

Norton, P. (ed.) (1996) National Parliaments and the European Union, London: Frank Cass.

O'Brennan, J. and Raunio, T. (eds.) (2007) National Parliaments within the Enlarged European Union, London: Routledge.

Peters, D., Wagner, W. and Glahn, C. (2014) 'Parliamentary control of CSDP: The case of the EU's fight against piracy off the Somali coast', European Security 23(4): 430-48.

Puntscher Riekmann, S. and Wydra, D. (2013) 'Representation in the European state of emergency: Parliaments against governments?' Journal of European Integration 35(5): 565-82.

Raunio, T. (1999) 'Always one step behind? National legislatures and the European Union', Government and Opposition 34(2): 180-202.

Raunio, T. (2000) 'Losing independence or finally gaining recognition? Contacts between MEPs and national parties', Party Politics 6(2): 211-23.

Raunio, T. (2009) 'National parliaments and European integration: What we know and agenda for future research', Journal of Legislative Studies 15(4): 317-34.

Raunio, T. and Hix, S. (2000) 'Backbenchers learn to fight back: European integration and parliamentary government', West European Politics 23(4): 142-68.

Raunio, T. and Wiberg, M. (2009) 'How to measure the Europeanisation of a national legislature?' Scandinavian Political Studies 33(1): 74-92.

Rosamond, B. (2000) Theories of European Integration, London: Palgrave Macmillan.

Saurugger, S. (2014) 'Europeanisation in times of crisis', Political Studies Review 12(2): 181-92.

Strelkov, A. (2015) 'Who controls national EU scrutiny? Parliamentary party groups, committees and administrations', West European Politics 38(2): 355-74.

Tweede Kamer. (2006) 'Parlement aan Zet: Voorstellen ter versterking van de parlementaire betrokkenheid bij Europa', http://www.eerstekamer.nl/id/vhyxhwl4tey0/document_extern/b21501_20 360_bijlage/f=/b21501_20_360_bijlage.pdf, accessed 18 October 2012.

van Keulen, M. (2012) At the workshop 'National Parliaments: Guardians of Democracy in the Building of a Closer European Union or Helpless Latecomers in the EU Decision-Making Process', The Hague, Campus of Leiden University, 20 September.

Winzen, T. (2012) 'National parliamentary control of European Union affairs: A cross-national and longitudinal comparison', West European Politics 35(3): 657-72.

\section{About the Authors}

Katjana Gattermann is Researcher at the Amsterdam Centre for Contemporary European Studies (ACCESS EUROPE). She is academic director of the Erasmus Academic Network on 
Parliamentary Democracy in Europe (PADEMIA) and co-editor of the LSE 'Europe in Question' Discussion Paper Series (LEQS). She is a scholar of European Union Politics and Political Communication. Her work feeds into debates about the legitimacy and accountability of politics within and across the European Union.

Anna-Lena Högenauer is Adjoint de Recherche at the Institute of Political Science at Luxembourg University. She holds a Ph.D. in Politics from the University of Edinburgh, and an M.A. in European Political and Administrative Studies from the College of Europe in Bruge. Her research interests include multi-level governance, multi-level parliamentarism and interest representation.

Ariella Huff received a Ph.D. in International Studies from Cambridge University in 2011, then worked for 3 years as a post-doctoral researcher for the Cambridge team of the Observatory of Parliaments after Lisbon Project. On the OPAL project, she specialised in parliamentary scrutiny of EU foreign and security policy. In 2014 she became a Specialist with the House of Commons Foreign Affairs Committee. 